\title{
Common bone and joint degeneration treatment by Chinese medicine
}

\section{Conceptual}

Just like all living organisms, the human body will degenerate over time. There are no exceptions with the only difference being when! Generally, the human body develops until 20years of age and then starts degenerating microscopically from about 30 years of age. Functionally, degeneration starts to appear at about 40years of age. From about 50years of age degeneration is clearly evident.

From 40years of age, degenerative changes to bones and joints occur due to hormone changes. Cartilage viscosity is reduced but more fibres are developed. Cartilage can be easily damage from impact as well as mechanical force received day by day over decades. Furthermore, osteological spurs develop. Also joints become inflamed and swollen (Tendonitis or Bursitis) due to irritation caused by calcification of ligaments and osteological spurs. This calcification can also affect the surrounding soft tissues and the inflammation can upset the periosteum therefore promote the development of spurs. This commences a vicious cycle.

Common bone and joint degeneration such as Cervical Spine Syndrome, Frozen Shoulder, and Disc Prolapsed of the Lumbar Spine, Osteoarthritis and Osteoporosis has shown poor treatment rates from Western medicine. On the other hand, Chinese medicine has shown a higher success rate in treating these problems with Chinese herbs (applied orally and externally), Tuina (a therapy that includes Chinese therapeutic massage, acupressure, spine and joint manipulation) and acupuncture. In some cases, combined therapy is required.

The author prefers Tuina for the first option to separate and loosen the muscular lumps or scar tissues, improve blood circulation, relax soft tissues and improve joint movement. As soon as the treatment course is completed, it can also reduce swelling and relieve pain.

Acupuncture is playing a great role in treating common bone and joint degeneration. Acupuncture can reduces swelling and relieves pain instantly. Scientific studies have showed that acupuncture has its pain relief mechanisms - one study showed that relevant meridians will trigger electromagnetic inductive effects in the fractal continuum of the neurovascular network.$^{1}$ In term of Chinese medicine, causes of pain vary: Congestion of blood, meridian obstruction, visceral weakness, or discord of Blood-Qi. In Chinese medicine, there is saying stating that "Where there is pain, there is a blockage." Blocks can be by stasis, qi deficiency or meridian blockage. Acupuncture can dredge the blockage and hence relieve the pain.

Herbal medicine used externally for bone and joint degeneration can treat directly to the lesion. It is recommended to apply an herbal

Volume 4 Issue 4 - 2016

Victor Wu
President \& Managing Director, Australian Chinese Medicine
Group Limited, Australia

Correspondence: Victor Wu, Waverley Road, Mount Waverley, Vic 3149, Australia, Tel 039802 I238,

Email victorwutem@yahoo.com

Received: June 02, 2016 | Published: November II, 2016 product, PainErase when there is pain and swelling. PainErase not only assists in the reduction of the inflammation of local soft tissues, reduction of swelling and relief of pain, it also can help to repair damaged soft tissues. The positive outcomes can be seen usually in the first or next day after application of PainErase.

If a patient with moderate to severe deficiency of Liver or/and Kidney funtion should consider taking Chinese herbal medicine. According to Chinese medicine theory, the bones are related to the kidney; ligaments and tendons are related to the liver. If there is a deficiency of kidney function, bones will lack supplement and become weaker. If there is deficiency of liver function, ligaments and tendons cannot be maintained and will lose strength. Therefore, invigoration of the kidney and the liver are the way to treat the causes of bone and joint degeneration. Assuredly to set up a formula, one must do so according to the principal rule of traditional Chinese medicine ---diagnosis and treatment based on an overall analysis of the illness and the patient's conditions ---- formulated individually.

For common bone and joint degeneration, treatment with Chinese medicine has far greater benefits than western medicine (Appendix).

\section{Acknowledgments}

None.

\section{Conflicts of interest}

Author declares there are no conflicts of interest.

\section{Funding}

None.

\section{References}

1. Chang S. The meridian system and mechanism of acupuncture - A comparative review. Part 2: Mechanism of acupuncture analgesia. Taiwan J Obstet Gynecol. 2012; Dec51(4):506-514. 